\title{
Transient focal neurological episodes - "amyloid spells": a TIA mimic that should be recognized
}

Episódios neurológicos focais transitórios - "amyloid spells": Um AIT mimic que deve ser reconhecido

Marx Lima de BARROS-ARAÚUO', Matheus Rocha de Seixas NOGUEIRA², Guilherme Marconi Guimarães Martins HOLANDA², Ícaro Araújo de SOUSA², Matheus Rodrigues CORREA², Pedro Jorge Luz Alves CRONEMBERGER²

A 74-year-old woman was admitted to our hospital with a history of episodes of left-sided hemiparesis and numbness for few minutes. A brain MRI showed cortical subarachnoid blood and diffuse cortical microbleeds, compatible with a diagnosis of cerebral amyloid angiopathy, according to modified Boston criteria ${ }^{1}$. Other possible causes of the MRI findings, such as coagulopathy and traumatic brain injury, were excluded.
Cerebral amyloid angiopathy, a common cause of lobar hemorrhage in older patients, can also present with transient focal neurological episodes, which are sometimes known as 'amyloid spells'. These are stereotyped, recurrent, transient episodes of numbness or weakness and may mimic a transient ischemic attack ${ }^{1,2,3,4,5}$.
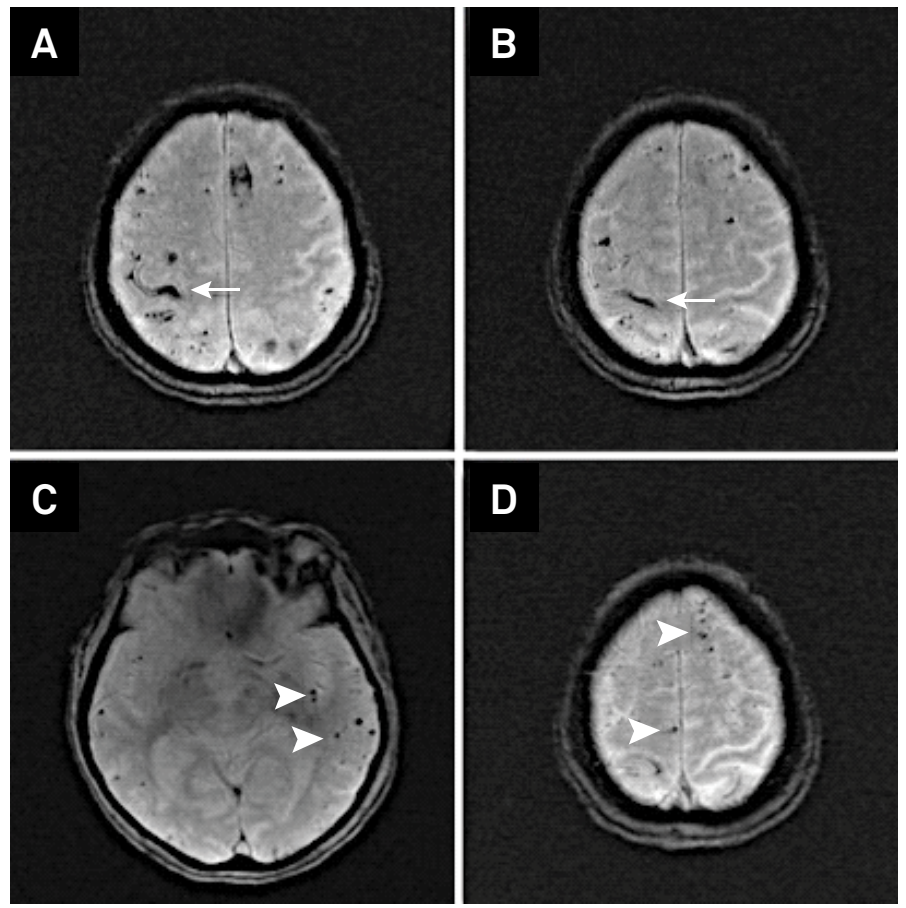

Figure. T2*-weighted gradient-recalled echo MRI of a 74-year-old woman who presented with transient neurological episodes. (A and B) Convexity subarachnoid hemorrhage and superficial cortical siderosis involving the right central sulcus (arrow). (C and D) Cortical microbleeds (arrowheads).'

\footnotetext{
'Universidade Federal do Piauí, Hospital Universitário, Teresina PI, Brasil;

${ }^{2} U$ niversidade Federal do Piauí, Faculdade de Medicina, Teresina PI, Brasil.

Marx Lima de Barros-Araújo (iD https://orcid.org/0000-0002-9943-1294; Matheus Rocha de Seixas Nogueira (iD) https://orcid.org/0000-0003-0644-5134; Guilherme Marconi Guimarães Martins Holanda (iD) https://orcid.org/0000-0003-3591-6847; Ícaro Araújo de Sousa (iD) https://orcid.org/0000-0002-9452-1207; Matheus Rodrigues Corrêa (iD https://orcid.org/0000-0001-9696-1786; Pedro Jorge Luz Alves Cronemberger (iD) https://orcid.org/0000-0002-5726-0546 Correspondence: Marx Lima de Barros Araújo; Hospital Universitário, UFPI; Campus Universitário Ministro Petrônio Portela, SG 07, Ininga; 64049-370 Teresina PI, Brasil; Email: marx.neuro@gmail.com

Conflict of interest: There is no conflict of interest to declare.

Received 23 February 2019; Received in final form 01 May 2019; Accepted 02 May 2019.
} 


\section{References}

1. Greenberg SM, Charidimou A. Diagnosis of cerebral amyloid angiopathy - Evolution of the Boston criteria. Stroke. 2018 Feb;49(2):491-7. https://doi.org/10.1161/STROKEAHA.117.016990

2. Pontes-Neto OM, Auriel E, Greenberg SM. Advances in our Understanding of the Pathophysiology, Detection and Management of Cerebral Amyloid Angiopathy. Eur Neurol Rev. 2012;7(2):134-9. https://doi.org/10.17925/ENR.2012.07.02.134

3. Charidimou A, Law R, Werring DJ. Amyloid "spells" trouble. Lancet. 2012 Nov;380(9853):1620.

https://doi.org/10.1016/S0140-6736(12)61333-6
4. Nadarajan V, Perry RJ, Johnson J, Werring DJ. Transient ischaemic attacks: mimics and chameleons. Pract Neurol. 2014 Feb;14(1):23-31. https://doi.org/10.1136/practneurol-2013-000782

5. Charidimou A, Peeters A, Fox Z, Gregoire SM, Vandermeeren Y, Laloux P, et al. Spectrum of transient focal neurological episodes in cerebral amyloid angiopathy: multicentre magnetic resonance imaging cohort study and meta-analysis. Stroke. 2012 Sep;43(9):2324-30. https://doi.org/10.1161/STROKEAHA.112.657759 